\section{A Junior Biology}

By Kenneth C. Sparrow. Pp. viii +187. (London : William Heinemann, Ltd., 1940.) $4 s$.

$\mathrm{T}$ HIS book is designed to cover a scheme of work for the first two years (of a five years course), thus leading up to the School Certificate years. In biology, of all subjects, it is extremely difficult to make a judicious selection of material which will provide a connected and correlated course; but the author has succeeded well. Yet, many teachers of biology would be ready to join issue with the author for considering seeds and seedlings and the development of young animals so early in the course. Though the logical thing to do, from the biological point of view, it is exceptionally difficult from the educational point of view, for children are not familiar with seeds and seedlings, neither are they with incubating eggs. They are, on the other hand, with mature plants and birds, and that is where many teachers would prefer to begin.

There are some attractive photographs in the book, but they seem to have been chosen too casually ; for example, Fig. 5, entitled "A Typewriter", shows more of a sturdy youngster than the machine it is intended to illustrate; the compression ignition engine cannot convey anything to a reader of such tender years, neither can the two excellent photographs of the development of the chick ; the diagram of a model of the lungs would convey more if two illustrations were given representing the two extreme positions; and it is always misleading to group several animals or plants together without indicating their relative sizes as is done in Fig. 29 where the octopus is indicated as being not much bigger than the snail.

The book is essentially practical in outlook, which is all to the good. It should satisfy those teachers who accept this type of course. The work is so designed as to stimulate the spirit of discovering things and finding out their meaning. This is very desirable since though it may be that the young reader will forget most facts stated in the book, he ought to leave it with a real desire to discover things biological for himself.

\section{Semimicro Qualitative Analysis}

By William Lloyd Evans, Alfred Benjamin Garratt and Laurence Larkin Quill. Pp. vii + 246. (Boston, New York, Chicago and London: Ginn and Co., 1940.) $10 s .6 d$. net.

$\mathrm{R}$ ECENT years have witnessed in inorganic chemistry the developments of a new technique involving analysis by semimicro methods, and the authors of "Semimicro Qualitative Analysis" have produced a most useful book for students in this field. A tremendous amount of detail, rendered more compact by an extensive system of abbreviations, has been compressed into this volume which includes five sections : an introduction describing apparatus, reagents and methods of procedure, one section each on the identification of cations and anions, a condensed outline of the theory of qualitative analysis, and finally a series of appendixes which is very comprehensive. The make-up of the book is somewhat novel in that it is of the loose-leaf variety, containing block outlines of methods, and flow sheets of experiments. It is therefore both a text-book and to some extent a laboratory note-book.

Semimicro analysis is not meant to displace the normal macroanalysis where larger quantities of materials are manipulated, but a course of this new technique supplies valuable training for a student since many of the rarer compounds are now, of necessity, analysed by micro methods.

The experimental details, which follow closely the better-known classical methods of analysis, are very clearly stated with ample explanations and directions. The theoretical part is couched in simple terms, but contains sufficient detail to enable the student to appreciate the methods employed in the experimental section. The appendixes will be found exceedingly useful since they contain much detailed instruction on the preparation of reagents, care of apparatus, general reactions of groups together with the usual tables of constants and lists of elements and symbols.

A course of microanalysis is almost essential now to aspiring chemists, and to such students this volume can be recommended with confidence as an indispensable item of their equipment.

\section{Practical Pharmaceutical Chemistry}

By F. N. Appleyard and Dr. C. G. Lyons. Fourth edition. Pp. vii + 174. (London: Sir Isaac Pitman and Sons, Ltd., 1939.) 6s. 6d. net.

THE fact that this text-book by Appleyard and Lyons has made its fourth appearance in ten years is sufficient evidence of its popularity. This new edition differs from its predecessors only by a slightly expanded chapter on qualitative organic analysis.

Since this work is intended for those students entering for the Chemist and Druggist Qualifying Examination, it is not exhaustive, in that the work necessary for the Preliminary Scientific Examination is only reviewed briefly. Instead attention is focused on more advanced topics. At the same time adequate instructions and explanations are given of the principles underlying analyses of pharmaceutical products, particularly volumetric analysis.

The fact that the experimental details are condensed to fit in with a special curriculum probably accounts for the fact that some of the sections, for example, those on the assay of alkaloids and on permissible adulterants, appear rather short. In those sections and in the chapters on gasometric and gravimetric methods, the authors have obviously selected specimen examples to illustrate the technique required. The sections on organic preparations and organic qualitative analysis are very well done, and the field covered should prove adequate for the purpose of pharmaceutical students.

The practical work described in the text-book forms a suitable complement to the theoretical studies necessary in this course. Undoubtedly this new issue will maintain the high popularity earned by previous editions. 